\title{
Una revisión sobre los Trastornos Espontáneos del Movimiento (TEM) en la esquizofrenia
}

\author{
Daniel Sotelo ${ }^{1}$, Romina Capellino², \\ María José Flores Moure ${ }^{3}$, Verónica Grasso ${ }^{3}$
}

1. Médico Psiquiatra, Hospital Neuropsiquiátrico Provincial de Córdoba.

2. Médica Psiquiatra, NEA, Neurociencias Aplicadas, San Carlos de Bariloche.

3. Médica, Posgrado de Psiquiatría del Hospital Neuropsiquiátrico Provincial de Córdoba.

Autor correspondiente: Daniel Sotelo, E-mail: danielgsotelo@gmail.com

\section{Resumen}

Los trastornos espontáneos del movimiento son observados con elevada frecuencia en la esquizofrenia. Conforman, junto con los síntomas negativos y cognitivos, aquellas manifestaciones más ligadas al deterioro de esta compleja condición, aunque son insuficientes para realizar el diagnostico. Cuando los síntomas psicóticos se expresan, estos fenómenos seguramente han estado generando consecuencias deletéreas durante años. Dentro de estos componentes intrínsecos, los trastornos motores son los más postergados, paradójicamente, como consecuencia del progreso de la farmacoterapia.

El tratamiento con antipsicóticos modificó la clínica de la esquizofrenia al producir mejorías en síntomas positivos, desorganización y agitación, pero también produjeron un cambio de otro orden, con los efectos colaterales que, muchas veces, ofrecen dificultad en ser distinguidos de la propia patología. Sobre todo, los neurolépticos comparten en mayor o menor medida la producción de síntomas extrapiramidales, expresados clásicamente en alteraciones motoras. Por ello, muchas veces se asocian, equivocadamente, estos movimientos anormales a la medicación antipsicótica de manera excluyente, ensombreciendo a aquellas manifestaciones motoras primarias.

El objetivo de este trabajo es destacar características, importancia y relación con la terapéutica convencional de los trastornos espontáneos del movimiento en la esquizofrenia.

Palabras clave: Esquizofrenia - Discinesias - Dopamina - Movimientos involuntarios anormales - Trastornos espontáneos del movimiento - Antipsicóticos. 


\title{
A REVIEW ABOUT SPONTANEOUS MOVEMENTS DISORDERS IN SCHIZOPHRENIA
}

\begin{abstract}
Spontaneous movements disorders are frequently observed in schizophrenia. Together with the negative and cognitive symptoms, they comprise manifestations more linked to the damage of this complex condition, although they are insufficient to make the diagnosis. When the psychotic symptoms express these phenomena, they have probably been causing deleterious effects for years. Within these intrinsic components, motor disorders are the most delayed, paradoxically, as a consequence of the progress of pharmacotherapy. Treatment with antipsychotics modified the clinical symptoms of schizophrenia by developing improvements in positive symptoms, disorganization and agitation, but also produced a change of another order, with the side effects that, many times, had difficulty in being distinguished from the pathology itself. Above all, the specific neuroleptics share to a greater or lesser extent of the production of extrapyramidal symptoms, classically expressed in motor alterations. Therefore, these abnormal movements are often mistakenly associated with antipsychotic medication in an exclusive manner, overshadowing primary motor manifestations.

The objective of this work is to highlight the characteristics, importance and relationship with the conventional therapy of spontaneous movements disorders in schizophrenia.
\end{abstract}

Keywords: Schizophrenia - Dyskinesias - Dopamine - Abnormal involuntary movements - Antipsychotics - Spontaneous movements disorders.

\section{Introducción}

Los trastornos motores en pacientes que padecen esquizofrenia son conocidos desde épocas previas a la introducción de los antipsicóticos (1). No obstante, este recurso terapéutico cambió la clínica de la enfermedad, tanto por sus efectos terapéuticos cuanto por sus efectos adversos. Temblor, rigidez, bradicinesia, trastornos espontáneos del movimiento (TEM) y otras alteraciones de la motilidad se asocian, muchas veces exclusivamente a los efectos secundarios de los fármacos utilizados para el tratamiento. Este es un concepto inexacto, ya que hay suficiente evidencia para considerar a los TEM como parte de la clínica de la esquizofrenia y esto puede tener implicancias teóricas, terapéuticas, pronosticas y medico legales.

Es cierto que el descubrimiento de los antipsicóticos además de un avance en el tratamiento significó un gran progreso en la investigación de la enfermedad. El conocimiento de su mecanismo de acción promovió la investigación sobre la fisiopatología, con la dopamina en el centro de la escena, puesto que todos los antipsicóticos son anti dopaminérgicos. Pero dentro de los "efectos adversos" de este progreso terapéutico se puede contar la apatía provocada hacia los trastornos motores no vinculados a la mediación.

El objetivo del presente trabajo es remarcar, cuáles son las características, la frecuencia, la importancia y el valor clínico, pronostico y conceptual de los trastornos motores en la esquizofrenia.

\section{Metodología}

Se utilizó como metodología la búsqueda bibliográfica en libros y tratados de la especialidad y en los registros de Medline, sin límite temporal. Se incluyeron artículos desde 1983 a 2017, en inglés y español. Se utilizaron palabras clave y búsquedas combinadas de palabras que involucran trastornos espontáneos del movimiento en esquizofrenia. Los términos de búsqueda que utilizados para recopilar la información incluyeron esquizofrenia, discinesias, discinesias espontaneas, parkinsonismo, dopamina, movimientos involuntarios anormales, trastornos espontáneos del movimiento, antipsicóticos, hitos tempranos del desarrollo motor.

\section{Dopamina y esquizofrenia}

Arvid Carlsson fue uno de los principales investigadores que aportó al desarrollo del conocimiento de la neurobiología de esta enfermedad. Demostró la presencia de dopamina en el cerebro, estableció su entidad como neurotransmisor y su distribución regional (principalmente en los ganglios de la base) y su función en la regulación de la actividad motora dentro del sistema extrapiramidal $(2,3)$.

Derivado de sus trabajos se postuló la "hipótesis dopaminérgica de la Esquizofrenia", aunque haya muchos otros factores involucrados. No obstante, no quedan dudas respecto de que la dopamina regula la actividad motora, en la esquizofrenia hay un desequilibrio en sus circuitos y todos los antipsicóticos son anti dopaminérgicos.

Se conoce que exceso de la actividad dopaminérgica se encuentra en la vía mesolímbica y se vincula a trastornos motores hipercinéticos y síntomas psicóticos. Paralelamente, se verifica una actividad disminuida en la vía mesocortical, sobre todo a nivel de la corteza prefrontal relacionada a síntomas negativos y cognitivos (4).

Con la llegada de los antipsicóticos se modificó la presentación de la enfermedad. Mejoraron los síntomas positivos y la agitación, pero agregaron sus efectos adversos. Sobre todo, los neurolépticos que comparten en mayor o menor medida la producción de síntomas extrapiramidales, expresados de manera manifiesta, aunque no exclusivamente, en la actividad motora del paciente. Aquí radica un problema frecuente, muchas veces se asocian, equivocadamente, estos movimientos anormales propios de la enfermedad, como consecuentes a la medicación antipsicótica de manera excluyente (5). 
Esto produce una subestimación que incluso alcanza al propio estudio del fenómeno. Los movimientos anormales involuntarios permanecen sub estudiados probablemente por ser largamente atribuidos a los antipsicóticos (6).

Tan natural es la asociación entre síntomas motores y medicación que mucha de la propia bibliografía especializada lo rubrica. Sobre discinesia tardía, por ejemplo: "Con este término se engloban una serie de movimientos anormales que aparecen tardíamente después de terapias con neurolépticos" (7).

Hasta el DSM 5 continúa clasificando al parkinsonismo y la discinesia tardía como trastornos del movimiento inducidos por la medicación (8).

\section{Trastornos espontáneos del movimiento}

Los síntomas extrapiramidales mayormente asociados a los neurolépticos son distonías agudas, parkinsonismo y discinesia tardía, pero varios déficits motores son observados en individuos con esquizofrenia, aun cuando nunca han sido medicados. Tales trastornos incluyen movimientos involuntarios anormales, softs neurological signs, síntomas catatónicos, retardo psicomotor y signos parkinsonianos $(9,10)$.

Los movimientos anormales vinculados a los antipsicóticos pero que con mayor frecuencia se presentan de forma independiente parecen ser el parkinsonismo y la discinesia tardía. Los reportes para otros movimientos como distonías agudas y acatisia son menos y los resultados no son concluyentes (11).

La discinesia se caracteriza por movimientos coreoatetoides involuntarios, con mayor frecuencia en la lengua, la boca o las extremidades y los síntomas parkinsonianos clásicos incluyen rigidez, bradicinesia y temblor.

\section{Estudio de los trastornos espontáneos del movi- miento}

Existen varias formas de estudiar la presencia espontanea de trastornos en el movimiento. Teniendo en cuenta las descripciones de autores previas a la introducción de los antipsicóticos y los estudios estadísticos de ese mismo periodo $y$, en el presente, a través de estudios de pacientes que no han estado expuestos a la medicación.

Dentro de las descripciones previas se encuentra Karl Kleist quien hizo un enorme desarrollo sobre lo que llamaba psicosis de la motilidad (12). Por otro lado, Khalbaum describió el síndrome catatónico en 1874 (13) y Kraepelin detalló trastornos motores en sus pacientes: "Los actos de nuestros pacientes a menudo terminan en formas cambiadas mórbidamente. Inclusive simples movimientos pueden manifestar cambios de esta clase. A veces, son llevados a cabo con un gasto demasiado grande de fuerza, o participan grupos innecesarios de músculos, o se utiliza una porción excesiva de los miembros, de modo que resultan sin gracia y torpes; o no son terminados, comienzan y terminan bruscamente y por lo tanto parecen rígidos, inexpresivos $y$ angulares. Otros pacientes también arriban a la meta del movimiento, no por el camino más corto, sino mediante rodeos, con todo tipo de cambios y de interpolaciones; agregan gestos ceremoniosos por los cuales el movimiento se vuelve antinatural, afectado y manierístico. [...] los procesos de respirar, hablar y escribir, estar parado y caminar, vestirse y desvestirse, dar la mano y comer, fumar, los gestos y el modo de ponerse a trabajar pueden ser influenciados y transformados en los modos más variado" (14).

Kraepelin expone una motilidad afectada por inexpresividad, rigidez, manierismos, estereotipias y atetosis.

Aún antes de los trabajos de estos autores clásicos, en un estudio retrospectivo de reporte de casos de pacientes con diagnóstico de esquizofrenia tratados entre $1850 \mathrm{y}$ 1889 , se documentan trastornos de movimientos similares a discinesia tardía en el 28\% de ellos (15).

Las discinesias espontáneas y el parkinsonismo han sido reportados en pacientes esquizofrénicos nunca medicados, y han sido indicados como una parte integral de la evolución de la enfermedad $(16,17)$. Lipton A, Cancro R. señalan que "Los movimientos coreoatetoides de los miembros e involuntarios de la región bucal pueden deberse a discinesia tardía, pero antes del advenimiento de los antipsicóticos se observaban en la esquizofrenia. El 10-25\% de los esquizofrénicos exhibe anomalías motoras no vinculadas con la medicación (18).

Hay suficiente evidencia para considerar que "una amplia variedad de anormalidades neurológicas ha sido reportadas en individuos con esquizofrenia quiénes nunca han sido tratados con medicación antipsicótica. Estos incluyen discinesias, parkinsonismo o signos extrapiramidales, signos neurológicos blandos y disminución de la percepción del dolor" (19).

\section{Trastornos espontáneos del movimiento, frecuen- cia}

Si la presencia es indudable, la frecuencia varía según los autores, llegando a reportarse cifras de presencia de síntomas en el 53\% de los pacientes nunca medicados $(20,21,22,23)$. La mayoría de los datos, menos tendientes a los extremos hablan de entre el 15 al 23\% de prevalencia de movimientos anormales (20).

Es importante remarcar lo que acontece en particular con la discinesia tardía, que normalmente alude a la provocada por la administración de antipsicóticos por períodos prolongados (24). No obstante, manifestaciones motoras indistinguibles de la discinesia tardía se observan en pacientes que nunca recibieron la medicación a la que se asocia, en una proporción que varía entre el 4 y el 11\% y que su presencia "particularmente la discinesia orofacial, parece ser parte de la presentación clínica de la esquizofrenia, sugiriendo que puede ser intrínseco a la fisiopatología de esa condición" (25).

\section{TEM de gravedad}

Uno de los aspectos destacados del estudio de los TEM es su valor clínico y predictivo, a través de la vincu- 
lación con ciertas formas clínicas. Su presencia se asocia a síntomas negativos, hebefrenia, deterioro cognitivo y curso crónico (20).

Esto tiene una connotación importante. Según esta perspectiva, algunas discinesias, sobre todo las orofaciales (fuertemente asociado a la medicación por su frecuencia y por su carácter clínico), podrían representar trastornos espontáneos del movimiento asociados a hebefrenia o formas deficitarias de esquizofrenia, con pronósticos más desfavorables.

Por otro lado, hay evidencia de que los pacientes con discinesia tardía tratados con neurolépticos tienen un mayor grado de desarrollo de síntomas negativos, disfunción cognitiva, signos neurológicos blandos que aquellos medicados, pero sin discinesia. Es razonable pensar que la emergencia de movimientos anormales, además de un efecto colateral de la medicación, puede también representar un trastorno motor asociado a la enfermedad o bien revelar una mayor predisposición a la aparición de tales trastornos del movimiento. Y, además, el riesgo de discinesia sería mayor en pacientes con síndrome deficitario (20).

En la clásica subdivisión de Crow de la esquizofrenia en dos síndromes, las discinesias tienen un valor destacado. El tipo I es a predominio de síntomas positivos, curso agudo, ausencia de deterioro intelectual y tiene una buena respuesta al tratamiento. El tipo II con síntomas negativos, curso crónico a estado defectual y posibilidad de deterioro intelectual, presenta pobre respuesta terapéutica (26). Las discinesias están más estrechamente relacionadas con las manifestaciones a largo plazo de la enfermedad que con su tratamiento, es muy posible que se los considere parte del síndrome de Tipo II (27) $y$, en ese sentido, pueden constituirse en un elemento semiológico de interés.

Dicho de otro modo, frente a un TEM siempre hay que tener en cuenta la posibilidad de que sea un componente propio de la enfermedad y un posible marcador clínico y evolutivo.

\section{TEM en el primer episodio}

La mayoría de la información sobre trastornos motores espontáneos derivan estudios de pacientes crónicos que no han estado expuestos a tratamiento. Pero también hay evidencia sobre lo que ocurre en el momento del primer episodio de pacientes que no han sido expuestos a antipsicóticos. La presencia de parkinsonismo oscila entre el 2 a 3\% (28) y el 27\% (29). La tasa de prevalencia mediana del parkinsonismo es del 17\%; es decir alrededor de 1 de cada 6 pacientes, una proporción similar a la reportada para los pacientes crónicos no tratados. La rigidez muscular es el signo parkinsoniano más común. La diada rigidez y bradicinesia, su tipo acinético, parece ser más frecuente que las manifestaciones no acinéticas, como el temblor, la sialorrea y el reflejo glabelar $(11,30)$. La tasa de prevalencia media de la discinesia espontánea es de 9\%. Variando entre márgenes de reporte del 0\% (31) al 14\% (32).
Pappa y Dazzan observaron que "La presencia de discinesia en pacientes con esquizofrenia constituye un fenómeno complejo y heterogéneo. Por lo tanto, algunos movimientos coreoatetoides anormales parecen ser intrínsecos a la patogénesis de la enfermedad, y ocurren espontáneamente incluso en las primeras etapas del proceso" (11).

En cuanto al parkinsonismo son interesantes los resultados obtenidos por Balda y cols. en una población del norte de nuestro país sin acceso a tratamiento. Como se esperaba, se encontró parkinsonismo en los pacientes que nunca habían realizado tratamiento (33), pero además se constató una mayor hiperecogenicidad de la sustancia negra, que constituye un marcador de parkinsonismo medicamentoso y que también se encuentra en enfermedad de Parkinson idiopática $(34,35)$. Este hallazgo podría delimitar un grupo de pacientes con mayor predisposición a síntomas negativos y un curso más severo (35).

Además, dentro de este grupo de pacientes con TEM con pronóstico menos favorable habría una mayor susceptibilidad a los efectos secundarios extrapiramidales de los antipsicóticos y mayor refractariedad del tratamiento (36). Por lo tanto, es necesario desarrollar herramientas que permitan su identificación, pues las dificultades en la evolución de este grupo de pacientes no se remiten solo a causas propias de la enfermedad, sino que, variables relacionadas con el tratamiento (como la elección del tratamiento farmacológico) podrían complejizar aún más sus perspectivas.

En resumen, la discinesia y el parkinsonismo son evidentes ya en el primer episodio de pacientes que no han sido expuestos a medicación. Esto nos permite pensar a estos trastornos como una disfunción subyacente a la enfermedad más que un proceso neurodegenerativo, fortaleciendo la hipótesis del componente neuromotor de la esquizofrenia. Es factible que la medicación, el envejecimiento y la neurodegeneración pueden contribuir aún más a su aparición o modificar su expresión.

\section{TEM y deterioro cognitivo}

La convivencia de síntomas motores y deterioro cognitivo ha sido observada pero la relación entre ambas no ha sido tan estudiada. Los pacientes con esquizofrenia que también presentan parkinsonismo realizan peores pruebas cognitivas que aquellos sin deterioro parkinsoniano, independientemente de la gravedad de la psicopatología, la sedación, la acatisia y la discinesia (37).

La presencia de parkinsonismo muestra fuerte asociación con déficits en memoria, funcionamiento ejecutivo y atención. En sujetos con psicopatología crónica y sin experiencia de tratamiento, así como también en familiares no afectados, la alteración motora parkinsoniana predice el deterioro cognitivo (38).

El impacto mayor de la esquizofrenia se encuentra en el área social e interpersonal y estas dificultades están estrechamente ligadas al deterioro cognitivo. Pero faltan indicadores clínicamente útiles y predictores de esta disfunción cognitiva. 
En este sentido, el parkinsonismo puede predecir de manera confiable la función cognitiva incluso en familiares no afectados. Estos datos pueden ser importantes a la hora de aportar elementos de valoración de riesgo para pacientes o familias y profundizar en el estudio de características clínicas de la enfermedad que permitan delimitar subtipos. Entre ellos la que se asocia con deterioro motor y deterioro cognitivo, que podría comenzar con las alteraciones de la motilidad y que entrañan un pronóstico más sombrío.

\section{TEM y riesgo aumentado de Discinesia Tardía}

Una consecuencia concreta y problemática del desconocimiento de la cualidad intrínseca de los TEM es tratarlos como efectos adversos de la medicación, habitualmente con anticolinérgicos como el biperideno o el trihexifenidilo,

El uso de anticolinérgicos puede conducir a un deterioro del curso de la enfermedad, al provocar síntomas cognitivos y aparición de delirios, con la agregada dificultad de tener que distinguir, una vez más, si estos son primarios o secundarios. Además, aunque el tema continúa siendo controvertido, el uso prolongado de anticolinérgicos en estos pacientes se podría asociar a lo que, en estos casos, conformaría un verdadero círculo vicioso, mayor predisposición a la aparición de discinesia tardía. En otras palabras, el tratamiento de las discinesias podría conducir a la aparición de más discinesias $(5,39$, $40,41)$.

\section{TEM como marcadores de riesgo familiar}

Los TEM pueden encontrarse en familiares de los pacientes que no padecen la enfermedad y, en ese caso, configurarse como un marcador de riesgo.

En este sentido, en un meta-análisis de 2010 se halló una fuerte asociación entre esquizofrenia con discinesia y parkinsonismo (como se esperaba), pero también resultados positivos para la presencia de estos trastornos motores en familiares de primer grado no enfermos (42).

De la misma forma que en los pacientes del norte de nuestro país con las anormalidades descriptas en la hiperecogenicidad en la sustancia nigra y el aumento del parkinsonismo, estas anomalías se observan en familiares de primer grado, aunque en menor medida (33).

Esto puede ser importante, ya que los trastornos del movimiento no solo representan un marcador evolutivo de la enfermedad, sino que pueden vincularse como marcador con el riesgo (genético) de desarrollar la enfermedad en personas que no la padecen.

\section{Hitos tempranos del desarrollo motor}

Una vía promisoria del estudio de la motilidad es la investigación del desarrollo motor y la posible presencia en el de marcadores de riesgo para el desarrollo de esquizofrenia. Esto supone la búsqueda de elementos presentes muchos años antes de la aparición de la psicosis que permiten vislumbrar algún tipo de vulnerabilidad remota.

En poblaciones de alto riesgo, se ha informado retraso en el desarrollo motor y destrezas motrices deterioradas en individuos que posteriormente desarrollaron esquizofrenia (43). En 1972, una muestra de 265 niños daneses de 11-13 años fueron filmados en condiciones estandarizadas mientras almorzaban. En 1991, el análisis de esas cintas demostró que aquellos niños que luego desarrollaron la enfermedad expresaron mayores déficits sociales y/o neuromotores (44). Asimismo, la observación de películas caseras filmadas por los padres durante la infancia de pacientes arrojó resultados similares (45). Y se hallaron también en estudios de cohortes en niños de 4 a 7 años que luego desarrollaron esquizofrenia (43).

Sin embargo, estos precursores neuromotores (por ejemplo, movimientos involuntarios de manos, brazos, sonrisa, movimientos oculares tipo nistagmus, elevación del codo, movimientos involuntarios de la cara) son sutiles y pueden carecer de especificidad suficiente para ser considerados (al menos por sí mismos) como marcadores para la prevención primaria (46).

Se han estudiado también los llamados hitos o logros tempranos del desarrollo motor tales como la deambulación, como predictores de desarrollo de esquizofrenia, aunque la revisión de estos no ha sido muy sistemática $(42,47,48)$.

Las variables de los hitos tempranos del desarrollo motor más estudiadas incluyen deambulación, pararse y sentarse sin apoyo, sostener la cabeza y tomar objetos con la mano. Hay otros hitos identificados como voltearse, tocar los pulgares con el dedo índice, rodar y gatear.

Los pacientes con esquizofrenia presentan un significativo retardo en lograr la deambulación, pararse y sentarse solos, comparados con controles (10).

Es decir, existiría una asociación entre retardo en deambular, pararse y sentarse sin ayuda como logros del desarrollo motor y el riesgo de esquizofrenia.

En un gran estudio de cohortes sobre la relación entre el retardo de los hitos tempranos de desarrollo motor y la esquizofrenia, con un seguimiento de 46 a 48 años a niños recién nacidos, contando con el registro parental, se reveló la asociación entre el retraso en el logro de los hitos del desarrollo temprano y la aparición posterior de la enfermedad. Los bebes que años después la manifestaron tendieron a sonreír, levantar la cabeza, sentarse sin apoyo, gatear más lejos, y caminar sin apoyo más tarde que los controles (48).

La evaluación de las anormalidades motoras tiene una serie de ventajas en relación con algunos otros posibles indicadores de riesgo. Pueden ser realizadas con bajo costo y buena fiabilidad en comparación con tecnologías como la resonancia magnética (44).

El valor predictivo de todos estos datos parece ser pequeño para identificar a sujetos de riesgo, pero dejan en evidencia cuán lejos están los síntomas psicóticos del probable comienzo de la enfermedad, impulsan a la búsqueda de posibilidades más precoces de diagnóstico y apoyan la teoría del trastorno del neurodesarrollo; es 
decir, una enfermedad neuroevolutiva de aparición secuencial que se basa en parte en estas alteraciones precoces en la motilidad de pacientes y familiares.

Existe una alta incidencia de anormalidades neuromotoras, conductuales e intelectuales muchos años antes de la eclosión de la enfermedad, lo cual señala un comienzo mucho más temprano de lo que indican los manuales para su diagnóstico (49).

La hipótesis del neurodesarrollo propone factores genéticos que predisponen, por ejemplo a una proliferación defectuosa o migración neuronal en el desarrollo del cerebro (50) pero incluye también factores de riesgo ambiental (ej. Influenza materna, desnutrición materna, parto en invierno y complicaciones del parto) que actúan antes del parto o perinatalmente (51), es decir muchos años antes de la psicosis.

Desde este marco, es posible sugerir un subtipo de esquizofrenia del neurodesarrollo que implica un deterioro infantil significativo y progresivo (52) que involucra anormalidades tempranas del lenguaje, del comportamiento y neuromotoras, inicio temprano y mal pronóstico que puede derivarse de la heterogeneidad en los factores etiológicos (43). Incluso dentro de estas disfunciones conductuales infantiles es posible que haya un subgrupo con problemas más graves e inicio más temprano. Este subgrupo también se caracteriza por mayores déficits neuromotores (53).

Queda aún por develar si este subtipo representa efectivamente un subgrupo de pacientes o bien el extremo de un continuo de riesgo (43).

Por otro lado, estos estadios precoces podrían ser objetivo de algún tipo de abordaje con el fin de disminuir la morbilidad. Aunque aún controvertida la posible intervención en una fase preclínica puede asociarse a resultados positivos que podrían incluir la mejoría sintomática, retardo en el inicio y posible prevención de la psicosis (44).

\section{Hitos tempranos y riesgo genético}

La evaluación de la motilidad temprana puede tener importancia, como se indicó, como indicadora de riesgo genético. El deterioro en las habilidades motoras en niñez ha sido caracterizado como un biomarcador para predecir el desarrollo de esquizofrenia en adultos $(54,55)$.

El estudio de habilidades motoras especificas en familiares de primer grado menores a 21 , que no padecen la enfermedad, tales como desarrollo de motricidad fina y gruesa, retraso en deambulación, deterioro en coordinación, movimientos anormales (temblores, tics, espasmos, movimientos atetoides), signos neurológicos blandos y habilidades motoras finas y gruesas muestran significativo deterioro (10). En resumen, las anomalías del movimiento observadas en la esquizofrenia también están presentes, aunque en menor medida, entre los miembros de la familia biológica, lo que fortalece la hipótesis de una probable base genética (56).

\section{Conclusiones}

Los trastornos motores como el parkinsonismo y la discinesia constituyen el componente motor de la esquizofrenia y la exclusiva asociación con el tratamiento ha ido en desmedro de su identidad clínica y han resultado subvalorados, pero su presencia espontánea debe siempre ser tenida en cuenta, ya que pueden encontrarse, incluso, al momento del primer episodio.

Además, su identificación es importante desde el punto de vista clínico ya que se vinculan a síntomas negativos, hebefrenia, deterioro cognitivo y cronicidad, formas evolutivas de pronóstico más desfavorable.

Los TEM pueden ser utilizados en la delimitación de subtipos dentro de la esquizofrenia. En la clasificación de Crow, las discinesias pertenecen al subtipo II, con pobre respuesta terapéutica, mayor deterioro, cronicidad y síntomas negativos.

Es relevante su valor predictivo. Ya sea en la pesquisa de marcadores de riesgo para personas que no padecen la enfermedad, pero que presentan vulnerabilidad genética (como ser familiares de primer grado de pacientes) o frente al retardo para la adquisición de logros de la motilidad, como riesgo de enfermar en el futuro.

En la búsqueda de biomarcadores de riesgo para la esquizofrenia la evaluación de los TEM puede aportar información con buena fiabilidad, obtenida en un examen clínico relativamente sencillo y de bajo costo.

Por último, los trastornos en la motilidad son marcadores de riesgo para el desarrollo de esquizofrenia y están presentes muchos años antes de la psicosis. Estos datos dejan en evidencia cuán lejos está el momento el diagnóstico de la enfermedad de su probable comienzo.

El retraso en el desarrollo motor y los precursores neuromotores fortalecen la hipótesis de patología del neurodesarrollo. Si bien son inespecíficos, su presencia, junto con otras manifestaciones prodrómicas como trastornos en la sociabilización o el déficit cognitivo, genera la inquietud de plantear intervenciones en pos de una mejoría sintomática y, quizás, del retraso o la prevención de la psicosis manifiesta.

\section{Conflictos de interés}

Los autores declaran no tener conflictos de interés respecto de este artículo. 


\section{Referencias bibliográficas}

1. Fenton WS. Prevalence of spontaneous dyskinesia in schizophrenia. Journal of Clinical Psychiatry. 2000; 61:10-14

2. Carlsson A. A Paradigm Shift in Brain Research. Science. 2001; 294: 1021-1024.

3. Carlsson A, Waters N, Holm-Waters S, Tedroff J, Nilsson M, Carlsson M. Interactions Between Monoamines, Glutamate, and GABA in Schizophrenia: New Evidence. Annu Rev Pharmacol Toxicol. 2001; 41:237-260.

4. De Erausquin G, Neurodesarrollo y Esquizofrenia. La paradoja de la teoría dopaminérgica: vino nuevo en odres viejos. Vertex, Rev. Arg. de Psiquiat. 2002, Vol. XIII: 189-197

5. Sotelo D, Diskinesias espontáneas en pacientes esquizofrénicos. Psicofarmacología, 34, 2005, 23-28.

6. Compton M, Fantes F, Ramsay Wan C, Johnson S, Walker E. Abnormal movements in first-episode, non affective psychosis: dyskinesias, stereotypies, and catatonic-like signs. Psychiatry Res. 2015 Mar 30; 226(1): 192-197.

7. Caponi R. Psicopatología y Semiología Pisquiátrica. Ed. Universitaria. 5a Edición. Santiago de Chile. 1998. Pag. 149

8. APA. Manual Diagnostico y Estadístico de los trastornos mentales (DSM-5), $5^{\circ}$ Ed. Arlington, VA: Asociación Americana de Psiquiatría, 2014.

9. Walther S, Strik W. Motor symptoms and schizophrenia. Neuropsychobiology. 2012;66(2):77-92.

10. Burton BK, Hjorthøj C, Jepsen JR, Thorup A, Nordentoft M, Plessen KJ. Research Review: Do motor deficits during development represent an endophenotype for schizophrenia? A meta-analysis. J Child Psychol Psychiatry. 2016 Apr;57(4):44656.

11. Pappa S, Dazzan P. Spontaneous movement disorders in antipsychotic-naïve patients with first-episode psychoses: a systematic review. Psychol Med. 2009 Jul;39(7):1065-76.

12. Kleist K (1997) Diez Comunicaciones. Introducción a las localizaciones cerebrales en neuropsiquiatria. Buenos Aires: Polemos, pp 207-228.

13. Berrios GE. 'The clinico-diagnostic perspective in psychopathology' by K. Kahlbaum. History of Psychiatry. 2007; $18: 233-245$

14. Kraepelin E (1996) La Demencia Precoz. 1o Edición en castellano. Buenos Aires: Polemos, pp 51-53.

15. Turner T. Rich and mad in Victorian England. Psychol Med. 1989;19: 29-44.

16. Peralta V, Campos MS, García de Jalón E, Cuesta MJ. Motor behavior abnormalities in drug-naïve patients with schizophrenia spectrum disorders. Movement Disorders Journal. 2010;25:1-9

17. Peralta V, Moreno-Izco L, Sanchez-Torres A, García de Jalón E, Campos MS, Cuesta MJ. Characterization of the deficit syndrome in drug-naïve schizophrenia patients: the role of spontaneous movement disorders and neurological soft signs. Schizophr Bull. 2014 Jan;40(1):214-24.

18. Lipton A, Cancro R (1995) "Esquizofrenia: Manifestaciones Clínicas", en Kaplan H, Sadock B. Tratado de Psiquiatría / IV. 6⿳亠丷厂 Edición. Buenos Aires. Intermédica, pp 906-916.

19. Fuller Torrey E. Studies of individual with schizophrenia never treated with antipsychotic medications: a review. Schizophrenia Research. 2002. 58;101-115.
20. Fenton WS, Jed R, Mc Glashan TH. Risk Factors for Sponaneous Dyskinesia in Schizophrenia. Archives of General Psychiatry. 1994; 51(8): 643-650.

21. McCreadie RG, Latha S, Padmavathi R, Ayankaran JR. Poor memory, negative symptons and abnormal movements in never. Treated Indian patients with schizophrenia. $\mathrm{Br} J$ Psychiatry 1997. 171;360-363.

22. Mc Creadi RG, Ohaeri JU. Movements disorders in never and minimally treated Nigeriran schizophrenic patients. $\mathrm{Br}$ J Psychiatry. 1994. 164; 184-189.

23. McCreadie RG, Padmavati R, Thara R, Srinivasan TN.Spontaneous dyskinesia and parkinsonism in never-medicated, chronically ill patients with schizophrenia: 18-month follow-up. Br J Psychiatry. 2002 Aug;181:135-7.

24. Van Kammen D, Marder S (1995) "Antagonistas de los Receptores de Dopamina", en Kaplan H, Sadock B. Tratado de Psiquiatría / IV. 6ํㅡㄹ Edición. Buenos Aires. Intermédica, pp 1901-1904.

25. Puri BK, Barnes TRE, Chapman MJ, Hutton SB, Joyce EM. Spontaneus dyskinesia in first episode schizophrenia. $J$ Neurolo Neurosurg Psyhiatry. 1999; 66:76-78.

26. Crow T, A Current View of the Type II Syndrome: Age of Onset, Intellectual Impairment, and the Meaning of Structural Changes in the Brain. Br J Psychiatry (1989), 155 (suppl. 7), 15-20.

27. Crow T, Owens D, JohnstoneE, Cross A, Owen F (1983) Does tardive dyskinesia exist? Modern Problems in Pharmacopsychiatry, 21, 206-219.

28. Chong SA, Subramaniam M, VermaS.Spontaneous parkinsonism in antipsychotic-naïve patientswithfirst-episodepsychosis. Can J Psychiatry. 2005 Jun;50(7):429-31.

29. Honer, WG, Kopala, LC, Rabinowitz, J Extrapyramida 1 symptoms and signs in first-episode, antipsychotic exposed and non-exposed patients with schizophrenia correlated psychotic illness. Journal of Psychopharmacology 2005, 19, 277-285.

30. Caligiuri MP, Lohr JB, Jeste DV. Parkinsonism in neuroleptic-naïve schizophrenic patients. American Journal of Psychiatry 150,1343-1348. 1993.

31. Chorfi, M, Moussaoui D. Never treated schizophrenic patients have no abnormal movements such as tardive dyskinesia. Encephale 11, 263-265. 1985.

32. Fenn DS, Moussaoui D, Hoffman, WF, Kadri, N, Bentouns, B, Tilane, et al Movements in never-medicated schizophrenics: a preliminary study. Psychopharmacology 123, 206210. 1996.

33. Kamis D, Stratton L, Calvó M, et al: Sex and laterality differences in parkinsonian impairment and transcranial ultrasound in nevertreated schizophrenics and their first degree relatives in an Andean population. Schizophr Res 2015; 164:250-255.

34. Bouwmans AE, Vlaar AM, Mess WH, et al: Specificity and sensitivity of transcranial sonography of the substantia nigra in the diagnosis of Parkinson's disease: prospective cohort study in 196 patients. BMJ Open 2013; 3:e002613.

35. Balda M, Calvó M, Padilla E, Guerrero G, Molina J, Florenzano $\mathrm{N}$, et al. Detection, Assessment, and Management of Schizophrenia in an Andean Population of South America: Parkinsonism Testing and Transcranial Ultrasound as Preventive Tools. Focus Vol. 13, No. 4, Fall 2015 
36. Smith RC, Kadewari RP, Rosenberger JR, et al: Nonresponding schizophrenia: differentiation by neurological soft signs and neuropsychological tests. Schizophr Bull 1999; 25:813-825.

37. Fervaha, G, Agid O, Takeuchi H, Lee J, Foussias G, Zakzanis $\mathrm{KK}$, et al. Extrapyramidal symptoms and cognitive test performance in patients with schizophrenia. Schizophr Res.2015. 161, 351-356.

38. Molina JL, González Alemán G, Florenzano N, Padilla E, Calvó M, De Erausquin G, et al. Prediction of Neurocognitive Deficits by Parkinsonian Motor Impairment in Schizophrenia: A Study in Neuroleptic-Naïve Subjects, Unaffected First-Degree Relatives and Healthy Controls From an Indigenous Population, Schizophr Bull. 2016 Nov; 42(6): 1486-1495.

39. KlawansL, Rubovits R. Effect of cholinergic and anticholinergic agents on tardive dyskinesia. I Neurolo Neurosurg Psyhiatry, 1974, 27, 941-947.

40. Bergman H, Soares-Weiser K. Anticholinergic medication for antipsychotic-induced tardive dyskinesia. Cochrane Database of Systematic Reviews 2018, Issue 1. Art. No.: CD000204. DOI: 10.1002/14651858.CD000204.pub2.

41. E, Novitch M, Alan Kaye D, Kata V, Kaye A. MedicationInduced Tardive Dyskinesia: A Review and Update Ochsner J. 2017 Summer; 17(2): 162-174.

42. Koning JP, Tenback DE, van Os J, Aleman A, Kahn RS, van Harten PN. Dyskinesia and parkinsonism in antipsychotic-naïve patients with schizophrenia, first-degree relatives and healthy controls: a meta-analysis. Schizophr Bull. $2010 ; 36(4): 723-31$.

43. Rosso IM, Bearden CE, Hollister JM, Gasperoni TL, Sanchez LE, Hadley T, Cannon TD: Childhood neuromotor dysfunction in schizophrenia patients and their unaffected siblings: a prospective cohort study. Schizophr Bull 2000; 26:367-378

44. Schiffman, J, Walker, E, Ekstrom, M, Schulsinger, F, Sorensen, H, Mednick, S Childhood Videotaped Social and Neuromotor Precursors of Schizophrenia: A Prospective Investigation. Am J Psychiatry 2004; 161:2021-2027

45. Walker EF, Savoie T, Davis D: Neuromotor precursors of schizophrenia. Schizophr Bull 1994; 20:441-451.
46. Isohanni M, Isohanni I, Koponen $H$, et al. Developmental precursors of psychosis. Curr Psychiatry Rep 2004; 6:168175.

47. Filatova S, Koivumaa-Honkanen H, Hirvonen N, Freeman A, Ivandic I, Hurtig T, et al. Early motor developmental milestones and schizophrenia: A systematic review and meta-analysis. Schizophr Res. 2017 Oct;188:13-20.

48. Sørensen HJ, Mortensen EL, Schiffman J, Reinisch JM, Maeda J, Mednick SA. Early developmental milestones and risk of schizophrenia: a 45-year follow-up of the Copenhagen Perinatal Cohort. Schizophr Res. 2010 May;118(1-3):41-7.

49. Harrison P.Schizophrenia: a disorder of neurodevelopment? Current Opinion in Neurobiology Volume 7, Issue 2, April 1997, Pages 285-289.

50. Torrey, E.F.; Taylor, E.H.; Bracha, H.S.; Bowler, A.E.; McNeil, T.F.; Rawlings, R.R.; et al. Prenatal origin of schizophrenia in a subgroup of discordant monozygotic twins. Schizophrenia Bulletin, 20(3):423-432, 1994.

51. Waddington JL: Schizophrenia: developmental neuroscience and pathobiology. Lancet 1993, 341531-536.

52. Foerster, A.; Lewis, S.W.; Owen, M.J.; and Murray, R.M. Low birth weight and a family history of schizophrenia predict premorbid functioning in psychosis. Schizophrenia Research, 5:13-20, 1991.

53. Neumann C, Grimes K, Walker E, Baum K. Journal of Abnormal Psychology 1995. Vol. 104. No. 4. 558-566

54. Erlenmeyer-Kimling L. Neurobehavioral deficits in of fspring of schizophrenic parents: liability indicators and predictors of illness. Am J Med Genet. 2000 Spring; 97(1):65-71.

55. Niemi LT, Suvisaari,JM, Haukka,JK, Lönnqvist JK. Childhood predictors of future psychiatric morbidity in offspring of mothers with psychotic disorder: results from the Helsinki High-RiskStudy. Br J Psychiatry. 2005 Feb;186:108-14.

56. Jeroen PF,Koning, Diederik E. Tenback, Jim van Os, André Aleman, et al. Dyskinesia and Parkinsonism in Antipsychotic-Naïve Patients With Schizophrenia, FirstDegree Relatives and Healthy Controls: A Meta-analysis. Schizophr Bull. 2010 Jul; 36(4): 723-731. 